\title{
The Ellipsoidal Surface Method Solving by Integration for Slope Stability Analysis
}

\author{
Sy Huu Pham ${ }^{1}$, Vinh Phu Pham ${ }^{2}$ and Minh Le Vu ${ }^{3}$ \\ 1. Thuy loi University, Hanoi 100803, Vietnam \\ 2. Delft University of Technology, Delft $2628 \mathrm{BW}$, the Netherlands \\ 3. Technology University of Braunschweig, Braunschweig 38448, Germany
}

\begin{abstract}
Landslide is common phenomena in construction field. Nowadays the cylindrical surface method has been used widely to analyze slope stability. However, this method contains two main disadvantages: firstly, the slip surface is assumed to be in circular shape while in fact it is ellipsoid commonly; and secondly, resolve by difference method causing immensely complicated process due to statically indeterminate equations. In this paper, the authors have presented a method that allows transferring of ellipsoid slip surface to cylindrical shape and carries out a single variable equation (by applying integration method). Also thanks to this method, the current cylindrical surface method has been proved that its application is proper for man-made slopes but usually inappropriate for natural ones. Those results help analysis of slope stability reaching closer to reality.
\end{abstract}

Key words: Slope stability, landslide, slice method, cylindrical surface method.

\section{Introduction}

Slop stability analysis plays an essential part on construction industry. There are a number of methods which have been introduced, such as the slice method of Refs. [1-4], simple circle method of Peterson [5], friction circle method of Taylor [6], inclined sliding surface method of Refs. [7, 8] and so forth. However the cylindrical surface method is convinced as the most appropriate solution in reality. Thus it is obvious why this method has been used worldwide. The method is based on an assumption of a circular slip surface, then divide the slip mass into vertical slices, weight those slices and taking into account components of tangential and normal forces and finally, derive a safety factor by evaluation of momentum equilibrium of the entire sliding mass:

$$
K=\frac{M_{c t}}{M_{g t}}=\frac{\sum W_{i} \cos \alpha_{i} \tan \varphi+\sum c l_{i}}{\sum W_{i} \sin \alpha_{i}}
$$

in which: $W_{i}$ is weight of slice $i, \alpha_{i}$ is inclination between slice $i$ base and horizontal plane, $l_{i}$ is length of slice $i$ base, $\varphi$ and $c$ are friction angle and cohesion of soil, respectively.

The common way to find out a slip surface with the lowest safety factor is to repeat the calculation changing position of slip center and slip radius. Despite its advantages, this method contains several drawbacks. For instance, it always considers slip surface as a circular one, while the fact shows that it is commonly in ellipsoid shape. Another concern is its complexity: for each slice, there are 3 variables, i.e. $W_{i}, \alpha_{i}$, and $l_{i}$; that means we have to deal with $3 n$ variables if the slip surface is divided into $n$ slices. A common solution for this complicated problem is the finite difference method which, unfortunately, leads to another difficulty: inaccuracy. Base of slices is a curve and because of difference method, calculating procedure considers it as a flat surface. In this paper, the authors introduce another approach of slope stability analysis which is able to overcome all of those shortcomings mentioned above.

Corresponding author: Pham Huu Sy, Dr., assoc. prof., research field: engineering geology. 


\section{Analysis of Ellipsoid Slope Stability Using Integration Method}

The traditional method considers slip surface as cylindrical shape and cuts out a slice to analyze stability. In reality, the largest cross-section is chosen to calculate. In addition, the slip surface is an ellipsoid, so the cross-section is overlay axis surface.

Fig. 1 illustrates the schematic cross-sections for calculation. A slip surface, which has potential as ellipsoid (in two-dimensional environment (2D) it is an ellipse as in Fig. 1a), can be determining using following equations:

$$
\begin{gathered}
\frac{X^{2}}{a^{2}}+\frac{Y^{2}}{b^{2}}=1 \\
Y=m X-p \\
Y=d \\
Y=h+d
\end{gathered}
$$

in which: $a, b$ are ellipse axis; $m$ is slope parameter; $d$ is distance from the horizontal surface to OX axis; $h$ is height of the slope.

By changing variables:

$$
X=\frac{\sqrt{a b}}{b} x ; Y=\frac{\sqrt{a b}}{a} y
$$

and

$$
\sqrt{a b}=R,
$$

those equations will become:

$$
x^{2}+y^{2}=R^{2}
$$

$$
\begin{gathered}
y=\frac{a m}{b} x-\frac{a p}{R} \\
y=\frac{a d}{R} \\
y=\frac{a(h+d)}{R}
\end{gathered}
$$

By this change in definitions, the ellipsoid slip surface is transformed into cylindrical shape (Fig. 1b). Divide slide mass into slices by a line which are described by equation: $x=i t$ ( $i$ is a counting number running from origin, $t$ is a constant increment), it $\leq R$ (Fig. 1). According to this method of dividing, there are 3 types of slices:

Type 1: Slices which have upper surface lying entirely on horizontal plane;

Type 2: Slices which have upper surface lying entirely on inclined plane;

Type 3: Slices which are located on edge or base of slide mass; have upper surface partly lying on horizontal and inclined plane.

Base of each slice with horizontal plane makes an angle $\alpha_{i}$. Slices have weight $W_{i}$ which can transform into two partial forces $T_{i}$ and $N_{i}$ :

$$
T_{i}=W_{i} \sin \alpha_{i} ; N_{i}=W_{i} \cos \alpha_{i}
$$

- Calculate slice's weight:

$$
W_{i}=\gamma \cdot S_{i}
$$

$S_{i}$ is slice's volume, in $2 \mathrm{D}$ environment it is calculated as:

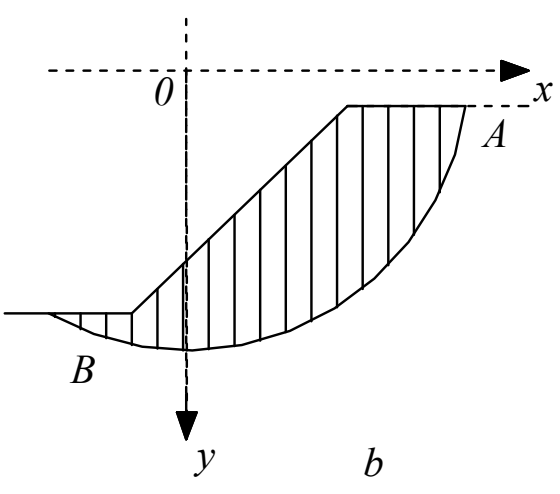

Fig. 1 Cross-section uses in calculation. (a) Original ellipsoid slip surface; (b) After transforming to circular. 
Type 1:

$$
\begin{aligned}
& S_{i(\text { base.horizontal })}=\int_{i t}^{(i+1) t}\left[-\frac{a(h+d)}{R}+\sqrt{R^{2}-x^{2}}\right] d x= \\
& -\frac{a(h+d) t}{R} \\
& +\frac{1}{2}\left[\begin{array}{l}
(i+1) t \sqrt{R^{2}-(i+1)^{2} t^{2}}+R^{2} \arcsin \frac{(i+1) t}{R} \\
-i t \sqrt{R^{2}-(i t)^{2}}-R^{2} \arcsin \frac{i t}{R}
\end{array}\right] \\
& S_{i(\text { top.horizontal })}=\int_{i t}^{(i+1) t}\left(-\frac{a d}{R}+\sqrt{R^{2}-x^{2}}\right) d x \\
& =-\frac{a d t}{R}+\frac{1}{2}\left[\begin{array}{l}
(i+1) t \sqrt{R^{2}-(i+1)^{2} t^{2}}+ \\
R^{2} \arcsin \frac{(i+1) t}{R}-i t \sqrt{R^{2}-(i t)^{2}} \\
-R^{2} \arcsin \frac{i t}{R}
\end{array}\right]
\end{aligned}
$$

Type 2:

$$
\begin{aligned}
& S_{i(\text { inclined })}=\int_{i t}^{(i+1) t}\left(-\frac{a m}{b} x+\frac{a p}{R}+\sqrt{R^{2}-x^{2}}\right) d x \\
& =-\frac{a m}{2 b}\left[(i+1)^{2} t^{2}-(i t)^{2}\right]+\frac{a p t}{R}+ \\
& \frac{1}{2}\left[\begin{array}{l}
(i+1) t \sqrt{R^{2}-(i+1)^{2} t^{2}}+R^{2} \arcsin \frac{(i+1) t}{R} \\
-i t \sqrt{R^{2}-(i t)^{2}}-R^{2} \arcsin \frac{i t}{R}
\end{array}\right]
\end{aligned}
$$

For slices of type 3, we can sort them on type 1 or type 2, depending on which part is larger. Because there are only two slices so error is fairly minor and able to be ignored.

\section{- Calculate length of slice base}

Length of each slice base is the length of curve between the two points: $x_{1}=i$ and $x_{2}=(i+1) t$. By using the equations: $x=R \cos \beta$ and $y=R \sin \beta$, the points $x_{1}$ will correspond with it where angle is $\beta_{i t}$ and $x_{2}$ will correspond with $(i+1) t$ and angle $\beta_{(i+1) t}$. The length of slice base calculates by following equation:

$$
\begin{aligned}
l_{i} & =\int_{\beta_{i}}^{\beta_{i+1}} \sqrt{(-R \sin \beta)^{2}+(R \cos \beta)^{2}} d \beta \\
& =R\left(\beta_{i+1}-\beta_{i}\right)
\end{aligned}
$$

in which:

$$
\beta_{i}=\arccos \frac{i t}{R} ; \beta_{i+1}=\arccos \frac{(i+1) t}{R}
$$

\section{- Calculate inclined angle of slice base}

In order to calculate this angle, we make derivative of mathematical function that present slice base in the middle point:

$$
\frac{d y}{d x}=\frac{x}{\sqrt{R^{2}-x^{2}}} \rightarrow \alpha_{i}=\arctan \frac{(i+1 / 2) t}{\sqrt{R^{2}-(i+1 / 2)^{2} t^{2}}}
$$

\section{- Calculate safety factor}

After collecting all those parameters, fill them in Eq. (1) and we will get safety factor of the slope. By applying this method, it requires ratio between the axes of the ellipse, and the challenged part is to carry out an appropriate ratio $(a / b)$ for each slide mass. In order to properly evaluate the ratio $a / b$, real investigations need to be implemented and then derive correlation between the ratio $a / b$ and soil condition.

According to this analysis it is convinced that the proposed method is a superior solution and overcomes shortcomings of the traditional circular arc method. More in details:

- The proposed method simulates slip surface in ellipse shape which is more realistic hence it comes up with more accurate output

With the traditional method, safety factor is calculated with assumption of cylindrical slip surface applying similarly for any type and condition of soil. The proposed method is more comprehensive for different types of soil. If soil is hard, $a / b=1$, and now the calculating procedures transform to circular arc condition. For the weaker type of soils, $a / b>1$, the problem turns to real ellipse condition. In such cases, if the traditional method is applied, the design slope will be calculated as Eq. (7), i.e. $(a / b) m$, while the slope is only stable with a safety factor of $m$ which is as in Eq. (3). This difference can be a threat to the construction. It also explains why application of the traditional method causes common failures: natural soils usually come up with high moisture, looseness; so slip surface 
mostly occurs in ellipse shape. This inappropriate application leads to a great number of failures in constructing of excavation pits, cut-off slope for roads and dam's abutments.

- Integration method can reduce complexity and provide a better result

As mentioned above, by applying difference method, the traditional method has a huge calculating work because it requires dealing with three variables: $W_{i}, \alpha_{i}$ and $l_{i}$, analyzing two partial forces $N_{i}$ and $T_{i}$ and then carrying out safety factor. In addition, it ignores curve shape of slice base but use plane base instead; so quantity of slices needs to increase in order to decrease inaccuracy. The proposed method use integration in calculation overcomes these issues. In more details, after integrating, each variable is transferred to a superlative formula of the single variable $R$. Thanks to it, calculating process becomes less complicatedly and at the same time, provides more accurate result due to taking consideration of curve part. Furthermore, $R$ can easily change to find $\mathrm{K}_{\text {minmin. }}$

- The proposed method allows a back-calculation solution to seek for reason of failure which is impossible with the traditional method

An important task of incident investigation is to pursue reason of failure. The traditional method and current commercial software only allow a conventional procedure: import input data, process and provide position of the slip surface that has the lowest safety factor. These numerical models have not support to import a particular slip surface to check its safety factor. The proposed method in this studying can help to clarify this issue. For a failure slope, minimum 2 boreholes are required to identify 2 points on the sliding surface $\left(X_{1}, Y_{1}\right)$ and $\left(X_{2}, Y_{2}\right)$, and accordingly the ellipse axis parameters $a$ and $b$ can be identified using Eq. (2). In combination with the parameters before sliding $m$ and $d$, the sliding surface can be identified close to its real situation, eventually the safety factor of the slope is obtained more accurately and helps to supply a better insight of the slope failure. In case of having more inspecting boreholes, the parameters $a$ and $b$ can be more accurately calculated by applying the minimum square method with the borehole results.

\section{Conclusions and Recommendations}

Those analysis above lead to the following conclusions:

- The slope in practice can slide in different forms, cylindrical or ellipsoid depending on soil state (compacted or natural soil) and conditions. Considering sliding surface to be cylindrical in all the case in the cylindrical surface method has shown the limitation of safety over-estimation and hinder the analysis and finding reasons of a slope failure;

- The proposed method using integration calculation on an ellipse has shown to closer approach a slope failure in practice and gives a reliable insight into the failure origination;

- The sliding slope dimension depends on the soil state and conditions. More studying on the dependence of the sliding surface axis ratio on the soil state parameters is recommended to improve the reliability of this proposed method and bring it into practice applications.

\section{Acknowledgments}

The authors thank Prof. Dr. Pho Duc Anh, Masters Tran Anh Ky and Nguyen Thi Vinh for giving suggestions.

\section{References}

[1] Bishop, A. W. 1955. "The Use of the Slip Circle in the Stability Analysis of Slopes." Geotechnique 5 (1): 7-17.

[2] Janbu, N. 1954. "Stability Analysis of Slopes with Dimensionless Parameters." Ph.D. thesis, Harvard University.

[3] Morgenstern, N. R., and Price, V. E. 1965. "The Analysis of the Stability of General Slip Surface." Geotechnique 15: 77-93.

[4] Spencer, E. 1967. "A method of Analysis of the Stability of Embankments Assuming Parallel Inter-slice Forces." Geotechnique. 17: 11-26.

[5] Peterson, K. E. 1916. "Kajraseti Goteborg des 5re Mars 
1916." TekniskTidskrift 46: 281-7, 289-94.

[6] Punmia, B. C., and Jain, A. K. 2005. "Soil Mechanics and Foundations." Firewall Media.

In Russian:
[7] Маслов, Н. Н. 1977. “Основы механика грунтов в инженерно-геологической пракике.” Мосва Недра.

[8] Шахунянц Г. М. 1953. “Земляное полотно железных дорог.” Мосbа. Трансжелдориздат. 